\title{
Hyperviscous Shock Layers and Diffusion Zones: Monotonicity, Spectral Viscosity, and Pseudospectral Methods for Very High Order Differential Equations
}

\author{
John P. Boyd ${ }^{1}$
}

Received March 17, 1993

\begin{abstract}
We solve two problems on $x \in[-\infty, \infty)$ for arbitrary order $j$. The first is to compute shock-like solutions to the hyperdiffusion equation, $u_{l}=(-1)^{j+1} u_{2 j, x}$. The second is to compute similar solutions to the stationary form of the hyperBurgers equation, $(-1)^{\prime} u_{2, x}+u u_{x}=0$; these tanh-like solutions are asymptotic approximations to the shocks of the corresponding time dependent equation. We solve the hyperdiffusion equation with a Fourier integral and the method of steepest descents. The hyper Burgers equation is solved by a Fourier pseudospectral method with a polynomial subtraction.

Except for the special case of ordinary diffusion $(j=1)$, the jump across the shock zone is described by nonmonotonic, uscillatory functions. By smearing the front over the width of a grid spacing, it is possible to numerically resolve the shock with a weaker and weaker viscosity coefficient as $j$, the order of the damping, increases. This makes such "hyperviscous" dampings very attractive for coping with fronts since, outside the frontal zone, the impact of the artificial hyperviscosity is much smaller than with ordinary viscosity. Unfortunately, both the intensity of the oscillations and the slowness of their exponential decay from the center of the shock zone decrease as $j$ increases so that the shock zone is much wider than for ordinary diffusion. We also examined generalizations of Burgers equation with "spectral viscosity", that is, damping which is tailored to yield exponentially small errors outside the frontal zone when combined with spectral methods. We find behavior similar to high order hyperviscosity.

We conclude that high order damping, as a tool for shock-capturing, offers both advantages and drawbacks. Monotonicity, which has been the holy grail of so much recent algorithm development, is a reasonable goal only for ordinary viscosity. Hyperviscous fronts and shock zones in flows with "spectral viscosity" are supposed to oscillate.
\end{abstract}

KEY WORDS: Hyperviscous; shocks; diffusion; shock capturing.

${ }^{1}$ Department of Atmospheric, Oceanic and Space Science, University of Michigan, 2455 Hayward Avenue. Ann Arbor, Michigan 48109. 


\section{INTRODUCTION}

In high Reynolds number flow, frontal zones or shocks form spontaneously. These fronts have widths small in comparison to any reasonable grid spacing. Roe (1986) reviews a variety of ingenious numerical schemes which cope with shocks without explicitly resolving them. An alternative, which is the only tactic discussed here, is to simply add sufficient artificial damping to smear out the frontal zone until the computational grid is sufficient to resolve it. Normally, two or three grid points in the region of high gradient is sufficient.

An alternative to ordinary viscosity is to employ a damping which is proportional to a higher derivative of $u(x)$ than the conventional second order viscosity or diffusion. Such "hyperviscosities" are very popular in geophysical modeling because the $2 j$ th derivative of $\exp (i k x)$ is $(-1)^{j} k^{2 j} \exp (i k x)$. Thus, the larger $j$ is, the smaller the effect of the damping on the low wavenumbers. At the same time, the damping increases with wavenumber so that the highest waves resolvable on the grid are strongly damped, insuring computational stability.

An obvious question is: how does hyperviscosity alter the structure of the shock? By means of multiple scales perturbation theory and matched asymptotic expansions, one can show that the frontal zone has its own self-similar dynamics where advection and diffusion reach a balance that is largely independent of the complexities of the flow outside the zone. The analysis is given in Boyd (1992), Blumen (1990a, b), Kevorkian and Cole (1981) and Lesser and Chrighton (1975).

To avoid drowning in detail, we shall limit ourselves to a simple theorem which is restricted to a generalized Burgers' Eq. (1.1).

\subsection{Matched Asymptotics Analysis of a Generalized Burgers' Equation with Variable Order Viscosity}

Theorem 1. The problem is

$$
u_{t}+u u_{x}+(-1)^{j} v^{2 j-1} u_{2 j x}=0 \quad u(x, 0)=Q(x)
$$

where the subscript " $2 j x$ " denotes the $(2 j)$-th derivative with respect to $x$ and where $v>0$. Then outside a narrow frontal layer of width $O(v), u(x, t)$ is well approximated by the solution of

$$
u_{t}+u u_{x}=0 \quad u(x, 0)=Q(x) \text { ["outer problem"] }
$$

The solution in the frontal zone is given by

$$
u(x, t) \sim s+J^{2 j-1} w(J X) \quad|X| \sim O(1)
$$


where $s(t)$ is the speed of the shock as given by the solution of the outer problem, i.e.,

$$
s(t)=\frac{1}{2}\left\{u\left(0^{-}, t\right)+u\left(0^{+}, t\right)\right\}
$$

$u\left(0^{-}, t\right)$ and $u\left(0^{+}, t\right)$ denote the values of the outer solution on either side of the shock and

$$
J(t)^{2 j-1}=\frac{1}{2}\left\{u\left(0^{-}, t\right)-u\left(0^{+}, t\right)\right\}
$$

$w(X)$ is the unique solution of the parameter free problem

$$
\begin{aligned}
(-1)^{j} w_{2 j X}+w w_{X} & =0 \quad x \in[-\infty, \infty] \\
\lim _{|X| \rightarrow \infty} w(X) & =\left\{\begin{array}{lc}
1 & X<0 \\
-1 & X>0
\end{array}\right.
\end{aligned}
$$

where the inner coordinate is

$$
X=\frac{x-\int^{\prime} s(\tau) d \tau+\phi}{v}
$$

where $\phi$ is a phase factor which is determined to lowest order by the requirement that $X=0$ at that value of $x$ where the shock first forms in the outer solution.

To render the local approximations spatially uniform, that is, valid both inside and outside the shock zone, it suffices to apply the inner approximation with $J(t)$ and $s(t)$ allowed to vary slowly with $x$ :

$$
\begin{aligned}
J(t)^{2 i-1} & =\frac{1}{2}\left\{u(x, t)-u\left(0^{+}, t\right)\right\} & & {[x<s(t)] } \\
J(t)^{2 j-1} & =\frac{1}{2}\left\{u\left(0^{-}, t\right)-u(x, t)\right\} & & {[x>s(t)] } \\
s(t) & =\frac{1}{2}\left\{u(x, t)+u\left(0^{+}, t\right)\right\} & & {[x<s(t)] } \\
s(t) & =\frac{1}{2}\left\{u\left(0^{-}, t\right)+u(x, t)\right\} & & {[x>s(t)] }
\end{aligned}
$$

The proof is given in Boyd (1992).

This theorem is quite remarkable because it implies that regardless of whether the hyperviscosity coefficient is large or small, regardless of whether there is one front or many fronts and regardless of the symmetry or speed of the front, the frontal zone is still described by a single function which is parameter-free once the order of the viscosity has been specified. Thus, we can answer our question about the effects of different orders of viscosity on the shock merely by solving Eq. (1.6) for various $j$.

Lest the restriction to the hyperBurgers equation seem too restrictive, note that Blumen (1990a, b) has demonstrated that Burgers equation, 
though it involves only a single spatial coordinate, is actually a consistent model for fronts in three-dimensional atmospheric frontogenesis. Boyd (1992) gives an even more general version of the theorem that incorporates an instability term: Even when the flow is amplifying in time, the shocks are still described by the solutions to Eq. (1.6).

Unfortunately, the hyperBurgers equation cannot be solved analytically. We offer two remedies. One is a polynomial-plus-Fourier pseudospectral method to solve Eq. (1.6) numerically for $j$ as large as 40 [80-th derivative damping]. The other is to examine the hyperdiffusion equation

$$
u_{t}=(-1)^{j+1} u_{2 j, x}
$$

We can solve this for arbitrary $j$ by a Fourier integral followed by the method of steepest descents, as explained in Sec. 2. We find that this equation has self-similar shocks which behave with increasing $j$ very much like the nonlinear solutions to Eq. (1.6).

Tadmor $(1989,1990)$ and Maday and Tadmor (1989) have introduced a still more general hyperviscosity, dubbed "spectral viscosity", which is chosen so that pseudospectral methods converge exponentially fast to the correct, high Reynolds solution away from the shock. (The shock itself has been smeared over a frontal zone of one or two grid points in width, so it is true of spectral viscosity, as of all artificial diffusion methods, that the frontal zone itself is too wide to faithfully approximate the shock of the real high Reynolds number flow, which by assumption is too narrow to explicitly resolve.) We explain why spectral viscosity also gives an oscillating frontal zone. Indeed, it appears that ordinary diffusion may be the only damping that gives monotonic shocks for Eqs. (1.1) and (1.6).

Lastly, hyperviscosity in an implicit consequence of high order difference methods. This is seen most easily in the "modified equation" analysis of Warming and Hyett (1974), Hedstrom (1975), and LeVeque (1990). For example, if a one-sided, first order upwind difference approximation is applied to the first derivative in the linear advection equation

$$
u_{t}+u_{x}=0
$$

then the usual error formula can be interpreted either as a first order approximation to the original equation, i.e.,

$$
u_{t}+\nabla u \sim u_{t}+u_{x}+O(h)
$$

where $\nabla$ is the difference operator and $h$ is the grid spacing or as a second order approximation to the modified partial differential equation

$$
u_{t}+\nabla u \sim u_{t}+u_{x}-\frac{1}{2} h u_{x x}+O\left(h^{2}\right)
$$


In words, Eq. (1.13) implies that the upwind difference has effectively added a weak diffusion term to the advection equation with a coefficient proportional to the grid spacing. Although Eq. (1.13) is nothing more than a restatement of the fact that the $O(h)$ error in the finite difference is proportional to the second derivative of the function being differentiated, the "modified equation" approach is of great conceptual value. For example, it explains why upwind differencing generates stable solutions even in the presence of shocks: the differencing effectively smears out the shock over a distance of $O(h)$. Similarly, a downwind differencing would change the sign in Eq. (1.13), creating an "anti-diffusion" which amplifies, rather than smoothes, giving instability no matter how accurately we approximate the time derivative.

The relevance of this perspective on differencing errors is that higher order upwind-biased schemes create similar artificial diffusion terms of the form

$$
h^{2 j-1}(-1)^{j} u_{2 j x}
$$

where $2 j$ is the number of grid points used in the approximation. The width of the resulting frontal zone is $O(h)$ because we can eliminate the factor of $h^{2 i}$ I by rescaling the $x$-coordinate via $x \Rightarrow X / h$. (Such a rescaling is implicit in the unit coefficients of Eqs. (1.6) and (1.8), for which the shock zone width is $O(1)$ when $t$ is $O(1)$.)

The question remains: What is the shape of a hyperviscosity-smoothed frontal zone when $j>1$ ? In the rest of this article, we offer some answers.

\section{THE HYPERDIFFUSION EQUATION}

The hyperdiffusion problem is

$$
\begin{gathered}
u_{t}=(-1)^{j+1} u_{2 j, x} \quad x \in[-\infty, \infty] \\
u(x, t=0)=\left\{\begin{array}{rc}
1 & x>0 \\
-1 & x<0
\end{array}\right.
\end{gathered}
$$

where $j$ is a positive integer and the subscript " $2 j, x$ " denotes the $2 j$ th partial derivative with respective $x$. The initial condition is chosen to be the signum function so that this linear equation will have solutions that mimic the shocks of its nonlinear generalization of Eq. (1.1) as closely as possible. It is easy to verify by direct substitution that the solution is 


$$
\begin{aligned}
& u(x, t)=A_{j}\left(\frac{x}{t^{1 /(2 j)}}\right) \\
& A_{j}(Z) \equiv \int_{0}^{z} \lambda_{j}(z) d z \\
& \lambda_{j}(x)=q_{j} \int_{-\infty}^{\infty} \exp \left(-k^{2 j}\right) e^{i k x} d k
\end{aligned}
$$

where $q_{j}$ is a proportionality constant chosen so that $A_{j}(\infty)=1$.

For the special cases of $j=1$ (ordinary diffusion) and $j \Rightarrow \infty$, the integrals can be evaluated analytically:

$$
\begin{aligned}
& A_{1}(Z)=\operatorname{erf}(Z / 2)=\frac{2}{\sqrt{\pi}} \int_{0}^{Z / 2} \exp \left(-y^{2}\right) d y \\
& \Lambda_{\infty}(Z)=\frac{2}{\pi} \operatorname{Si}(Z)=\frac{1}{\pi} \int_{0}^{Z} \frac{\sin (y)}{y} d y
\end{aligned}
$$

The limit $j \Rightarrow \infty$ follows from

$\lim _{j \rightarrow \infty}\left(\exp \left[-z^{2 j}\right]\right)=\left\{\begin{array}{l}1|z|<1 \\ 0|z|>1\end{array} \Rightarrow \lambda_{\infty}(z)=q_{\infty} \int_{-1}^{1} e^{i k z} d k=2 q_{\infty} \frac{\sin (z)}{z}\right.$

Figure 1 compares these two limiting cases (solid) with the solutions for fourth and sixth derivative damping. The error function in Eq. (2.4) varies smoothly and monotonically from -1 to 1 .

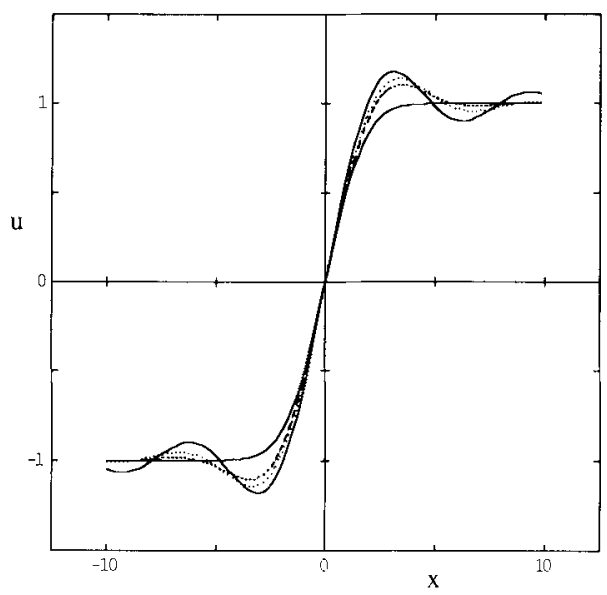

Fig. 1. Shocks for the ordinary diffusion equation. Solid monotonic curve: $\boldsymbol{A}_{1(x)}$ (ordinary diffusion). Dashed (smallest oscillations): $A_{2}$ [fourth derivative damping]. Dotted (medium oscillations): $A_{3}$ [sixth derivative damping]. Solid oscillatory curve (largest oscillations): $A_{\infty}$ (Sine integral). 
Furthermore, it asymptotes very quickly to its limits as $|x| \rightarrow \infty$ with the $\left|\Lambda_{2}(x)\right|-1$ decreasing as $\exp \left(-x^{2} / 4\right)$.

However, the sine integral in Eq. (2.5) is just the opposite: It oscillates, and the oscillations decay very slowly as $O(1 / x)$. Its asymptotic expansion is (Abramowitz and Stegun, 1965):

$$
\frac{2}{\pi} \operatorname{Si}(x) \sim 1-\frac{2}{\pi} \frac{1}{x} \cos (x)+O\left(1 / x^{2}\right) \quad x \gg 1
$$

The fronts for $j=2$ and $j=3$ are intermediate between these extremes. As $j$ increases, the amplitude of the largest crest increases, but is bounded by the maxima of the sine integral. A more significant change is that the oscillations decay more slowly in $|x|$ for larger $j$. Thus, although the neighborhood of the center of the front is little changed as $j$ increases - note that the four graphs in Fig. 1 fall atop one another for $|x|<0.5$ - the width of the oscillatory region expands with increasing $j$ until the sine integral is obtained in the limit.

By using the method of steepest descents (Bender and Orszag, 1978, and Boyd, 1982), we can approximate the shocks for general $j$. (We first approximated $\lambda_{j}(x)$ and then employed Laplace's method, which is steepest descent for the special case for which the dominant part of the integral is at an endpoint, to approximate $A_{j}(\infty)-A_{j}(x)$.) For fourth derivative damping $(j=2)$,

$$
A_{2}(x) \sim 1+1.15 \frac{1}{x^{2 / 3}} \exp \left(-\sigma x^{4 / 3}\right) \cos \left\{\sigma \sqrt{3} x^{4 / 3}-\frac{5}{6} \pi\right\} \quad x \rightarrow \infty
$$

where $\sigma=(3 / 8) 2^{-2 / 3}=0.236$. The oscillations decay exponentially, but the rate of decay is slower than the $\exp \left(-x^{2}\right)$ decay in the error function. Figure 2 compares the asymptotic approximation in Eq. (2.8) with the exact solution and displays the error. Except very close to the origin, the steepest descent approximation is very good and becomes better with increasing $x$.

Only positive $x$ is shown because $\Lambda_{j}(-x)=-\Lambda_{j}(x)$ for all $x, j$. Equation (2.8) may be extended to all $x$ by multiplying the R. H. S. by $\operatorname{sgn}(x)$.

The complete asymptotic approximation for general $j$ is rather messy, but the crucial point is, that ignoring algebraic factors of $x$ (i.e., $x^{-2 / 3}$ for $j=2$ ) and multiplicative constants,

$$
A_{2}(x)-1 \sim[] \exp \left(-\mu x^{2 j /(2 i-1)}\right) \cos \left\{\gamma x^{2 / /(2 j-1)} \text { - phase }\right\} \quad x \rightarrow \infty
$$




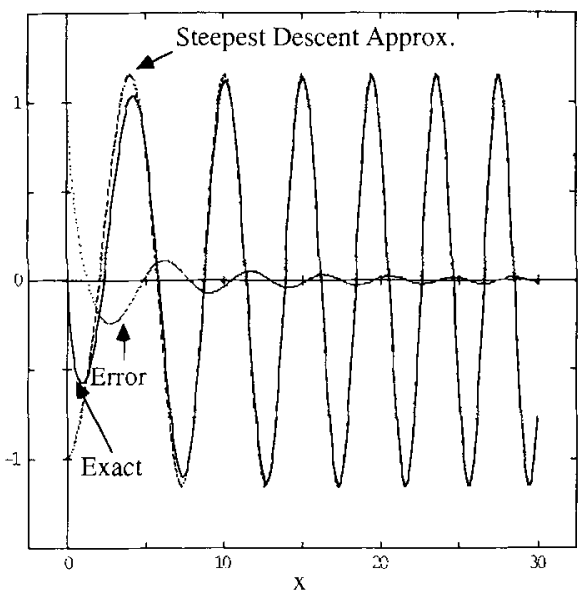

Fig. 2. The error in the steepest descent approximation of Eq. $(2.8)$ to the front for the hyperdiffusion equation with fourth derivative damping. All curves display the scaled function $\Psi=\left[A_{2}(x)-1\right] x^{2 / 3} \exp \left(\sigma x^{4 / 3}\right)$. Solid: exact $A_{2}(x)$. Dashed: steepest descent approximation of Eq. (2.8). Dotted: Absolute error.

where [ ] denote an algebraic factor of $x$ and "phase" denotes a phase constant and

$$
\begin{aligned}
& \mu=(2 j-1)\left(\frac{1}{2 j}\right)^{2 j /(2 j-1)} \sin \left(\pi \frac{1}{2(2 j-1)}\right) \\
& \gamma=(2 j-1)\left(\frac{1}{2 j}\right)^{2 j /(2 j-1)} \cos \left(\pi \frac{1}{2(2 j-1)}\right)
\end{aligned}
$$

The arguments of the exponential and the cosine are identical except for the trigonometric functions in Eq. (2.10). The crucial point is that in the limit $j \Rightarrow \infty, \mu \Rightarrow 0$ while $\gamma \Rightarrow 1$. Thus, the exponential decay disappears, leaving only the sinusoidal factor to oscillate with unit wavenumber, modulated by an algebraic factor of $x$. This is all perfectly consistent with the known asymptotic behavior of the sine integral, $\mathrm{Si}(x)$, which is the limit $j \Rightarrow \infty$.

These results present us with a paradox. Increasing the order of the damping is a Good Thing in the sense that smaller wavenumbers are damped less and less as $j$ increases. This in turn implies less and less damping in smooth regions away from the front. But paradoxically, increasing the order of the damping is also a Bad Thing because the front becomes more and more oscillatory and the wiggles extend farther and farther away from the front. 
The ultimate high order damping is a "band-limited" diffusion: All wavenumbers below the bandwidth $W$ are not damped at all while all wavenumbers larger than $W$ are truncated. The result is that the front assumes the shape of the sine integral. But $\mathrm{Si}(x)$, as shown in Korner (1988) and other Fourier texts, is the asymptotic approximation to the Gibbs Phenomenon, that is to say, to the wiggles that develop when the Fourier series (or integral) of a discontinuous function is truncated. (For this reason, we shall dub the $\mathrm{Si}(x)$ solution the "Gibbs' Shock" in later sections.) The Gibbs Phenomenon is generally regarded as a Very Bad Thing. In the remaining sections, we show that this ironic equation

$$
\text { Higher Order Damping } \Leftrightarrow \text { Wider Wiggles }
$$

also applies to the hyperBurgers Eq. (1.6) and to its spectral viscosity generalizations.

One important point is that in the steepest descent method, the exponential and trigonometric factors come from computing the stationary points of the phase function of the integral and then substituting these into the phase function. For example, if we generalize power-law diffusion by writing the diffusion equation after Fourier transformation as

$$
\begin{aligned}
& U(k, t)_{t}=-Q(k) U(k, t) \\
& u(x, t) \equiv \int^{\infty} U(k, t) e^{i k x} d k
\end{aligned}
$$

(where $Q(k)=k^{2 j}$ for Eq. (2.1)), then the formal solution is

$$
u(x, t)=\int_{-\infty}^{\infty} U(k, 0) \exp (-Q(k) t+i k x) d k
$$

Define the phase function

$$
\varphi(x, t, k)=-Q(k) t+i k x
$$

The steepest descent approximation is a sum of all stationary points on the deformed contour of integration of the form

$$
\left.u(x, t) \sim \sum_{\substack{\text { all stationary } \\ \text { points } s}} U\left(k_{s}, 0\right) \text { [algebraic factor }\right] \exp \left(\varphi\left(k_{s}[x, t]\right), x, t\right)
$$

where the stationary points $k_{s}(x, t)$ are the roots of the first derivative of the phase function, i.e., solve

$$
\frac{d Q(k)}{d k}=i \frac{x}{t}
$$


Because of the factor of $i$ in Eq. (2.16), in general all the stationary wavenumbers are complex, and so usually is $\phi\left(k_{s}\right)$. Consequently, the normal, expected behavior of a shock-like solution to a generalized diffusion equation is that the frontal zone is oscillatory.

The case $j=1$, i.e., $Q(k)=k^{2}$, is very special. The only stationary point is pure imaginary $\left(k_{s}=i x /(2 t)\right)$ and $\exp \left(\phi\left[k_{s}\right]\right)=\exp \left(-x^{2} / 2 t\right)$, which is purely real. But all other power law viscosities and general $Q(k)$ will give a complex argument to the exponential, which implies that at least for large $x, u(x, t)$ is not monotonic but oscillatory.

\section{A POLYNOMIAL-FOURIER PSEUDOSPECTRAL METHOD FOR COMPUTING TANH-LIKE SOLUTIONS}

When the solution $u(x)$ to a problem on an infinite interval decays exponentially to 0 as $|x| \Rightarrow \infty$, Fourier domain truncation is a standard method. The problem is solved by a Fourier series on the truncated domain $[-L, L]$ with boundary conditions of periodicity with period $2 L$ (Boyd, 1988).

We can understand the success of both Fourier domain truncation and also the polynomial-Fourier generalization needed here by examining the "Fourier Asymptotic Coefficient Expansion" (FACE). Since all the solutions discussed here are antisymmetric with respect to the origin (i.e., $f(x)=-f(-x)$ for all $x)$, it suffices to specialize to a Fourier sine representation.

Let

$$
f(x)=\sum_{n=1}^{\infty} b_{n} \sin \left(\frac{n \pi x}{L}\right)
$$

Then the Fourier coefficients have the usual integral representation

$$
b_{n}=\frac{2}{L} \int_{0}^{L} f(x) \sin \left(\frac{n \pi x}{L}\right) d x
$$

Repeated integration-by-parts gives

$$
\begin{aligned}
b_{n}= & \frac{2}{\pi}(-1)^{n+1} \sum_{m=0}^{M}(-1)^{m}(L / \pi)^{2 m} \frac{f^{(2 m)}(L)}{n^{2 m+1}} \\
& +\frac{2}{\pi}\left(\frac{L}{\pi}\right)^{2 M+1} \frac{(-1)^{M+1}}{n^{2 M+2}} \int_{0}^{L} f^{(2 M+2)}(x) \sin \left(\frac{n \pi x}{L}\right) d x
\end{aligned}
$$


Equation (3.3) is exact for any $M$; the $M$ th order FACE is obtained by dropping the integral term to achieve an error which is $O\left(1 / n^{2 M+2}\right)$ in the limit $n \Rightarrow \infty$ for fixed $M$ (Lyness, 1971, 1984, and Boyd, 1989).

If $f(x)$ is a periodic, infinitely differentiable function, we can integrateby-parts an arbitrary number of times. If the boundary terms in the series in Eq. (3.3) vanish, the Fourier coefficients must decrease faster than a finite inverse power of $n$. This rate of convergence is variously labelled "infinite order", "exponential" or "spectral" (Boyd, 1989).

Unfortunately, when domain truncation is used, the true solution is not periodic and the boundary terms do not vanish. However, if $f(x)$ is decaying exponentially fast as $|x|$ increases, then for sufficiently large $L$, the boundary terms in Eq. (3.3) are exponentially small. A Fourier pseudospectral algorithm will then give superb accuracy as shown in (Boyd, 1988).

The complication here is that the shocks for the hyperBurgers Eq. (1.6) and for the hyperdiffusion Eq. (2.1) are "tanh-like", that is to say, asymptote to different values as $x$ tends to positive and negative infinity. (In fact, the Burgers shock for $j=1$ is $\tanh (x / 2)$ !) The FACE shows that if we expand $\tanh (x)$ itself on a large interval as a Fourier sine series, the coefficients are, using $f(L) \sim 1$,

$$
b_{n} \sim \frac{2}{n \pi}(-1)^{n+1} \quad n \gg 1
$$

The reason for this pitiful rate of convergence is that the sum $f_{S}(x)$ of the Fourier sine series is discontinuous, jumping from 1 to -1 at $x=L$ so that $f_{S}(L)=-f_{S}(-L) \sim-1$ as required by the $(2 L)$-periodicity of the sines.

However, the remedy is easy. First, note that the tanh function asymptotes exponentially fast to its asymptotic value, i.e.,

$$
\tanh (x)-1 \sim 2 \exp (-x) \quad x \gg 1
$$

Thus, all the higher derivatives in the FACE series do decrease exponentially fast with $L$. Only the leading $(m=0)$ term in the FACE series is a problem.

We can eliminate the problem by defining

$$
f(x) \equiv u(x)-x / L
$$

The new unknown $f(x)$ asymptotes to zero. Consequently, it has a rapidly convergent sine series and Eq. (3.4) is irrelevant.

Thus, our numerical method is to replace the unknown $u(x)$ by $f(x)$ via Eq. (3.6) and then apply the usual Fourier pseudospectral method (Boyd, 1989) to compute $f(x)$. With this modified procedure, we have 
solved the hyperBurgers' shock Eq. (1.6) for $j$ as high as 40 , that is, solved a differential equation of 80 th order.

Three minor technical points remain. First, the $x$-plus-Fourier series used to make Fig. 1,

$$
A_{j}(x)=x / L+q_{j} \sum_{n=1}^{\infty} \frac{\exp \left(-[n \pi / L]^{2 j}\right)}{n} \sin \left(\frac{n \pi x}{L}\right)
$$

is a legitimate solution to the diffusion equation for all $x$, albeit a rather peculiar one whose graph resembles stairsteps, and not merely an approximation to the infinite interval solution which is accurate on $[-L, L]$. In contrast, the $x$-plus-Fourier series for $\tanh (x)$ or a solution to the hyperBurgers' shock equation is a representation valid only on $x \in[-L, L]$. This limited range of validity does not alter the fact that the $x$-plus-Fourier series has only exponentially small error in approximating the infinite interval shock for $x \in[-L, L]$.

The second technical point is: How large should $L$ be? The answer is that $(u(L)-1)$ should be smaller than the desired error tolerance. Unfortunately, because the oscillations decay more and more slowly as $j$ increases, $L$ must increase with $j$. In practice, we had no trouble with $j$ as large as 40 even on a microcomputer.

The third issue is: How many Fourier sines do we need? As for the hyperdiffusion equation, the solutions to Eq. (1.6) turned out to be more and.more bandlimited as the damping order $j$ increased. That is, all coefficients $n$ such that

$$
\frac{n \pi}{L}>1
$$

tend rapidly to zero as $j$ increases. Because the hyperBurgers equation is quadratically nonlinear, the Fourier series for the nonlinear term has twice the bandwidth of $u(x)$ itself. So, for large $j$, we obtained excellent results by taking the truncation of the sine series to be

$$
N>2 L / \pi
$$

For the solutions to the 80th order $(j=40)$ version of Eq. (1.6), for example, we found by varying $L$ and $N$ that we obtained reproducible graphs with $L=80 \pi$ or larger and $N=200$. 


\section{SHOCKS IN THE HYPERBURGERS EQUATION}

The problem is Eq. (1.6):

$$
\begin{array}{cc}
(-1)^{j} u_{2 j, x}+u u_{x}=0 & x \in[-\infty, \infty] \\
\lim _{|x| \rightarrow \infty} u(x) & =\left\{\begin{array}{rc}
1 & x<0 \\
-1 & x>0
\end{array}\right.
\end{array}
$$

The only known analytical solution is for $j=1$ (ordinary, second derivative diffusion):

$$
u(x)=-\tanh (x / 2) \quad[j=11
$$

The case $(j=2)$ is a special case of the Kuramoto-Sivashinsky equation (Hooper and Grimshaw, 1988), but this and all other cases including the limit $j \Rightarrow \infty$ have defied analytical solution.

The tanh function is qualitatively like the error function, which is its counterpart for the hyperdiffusion equation, in that (i) it varies monotonically between its limits of \pm 1 and (ii) decays exponentially fast to those limits with increasing $|x|$. However, the error function decays like a Gaussian whereas the tanh function decays like $\exp (-x)$ : Even for $j=1$, the diffusive and Burgers shocks are different, though similar.

The numerical solutions show that the same is true for other $j$. The cases $j=1,2$, and 3 are compared in Boyd (1992). Figure 3 compares $j=5$, 10,20 and 40 on graphs of three different widths. Oscillations damp out with $|x|$ more slowly as $j$ increases. In Fig. 3a, for example, the difference between the second crest and the asymptotic value, -1 , is more than twice as large for $j=40$ as for $j=20$. In Fig. 3b, oscillations are visible for $j=40$ even for $x=250$, but the wiggles in the $j=5$ shock are invisible beyond $x=50$.

This behavior is consistent with an asymptotic analysis of the hyperBurgers equation for large $x$ where the equation reduces to

$$
(-1)^{j} u_{2 j . x}-u_{x}=0 \quad x>0
$$

This constant coefficient differential equation is easily solved; the slowest decaying component is of the form

$$
\begin{gathered}
u \sim \exp (-\mu x) \cos (\gamma x-\text { phase }), \quad x \rightarrow \infty \\
\mu=\sin \left(\pi \frac{1}{2(2 j-1)}\right) \\
\gamma=\cos \left(\pi \frac{1}{2(2 j-1)}\right)
\end{gathered}
$$


(a)

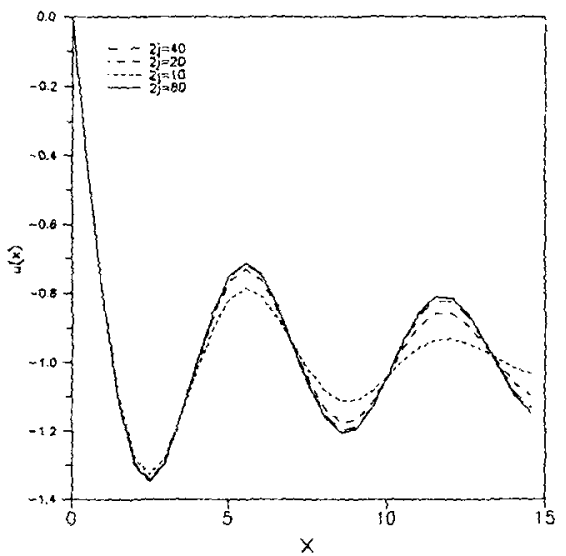

(b)

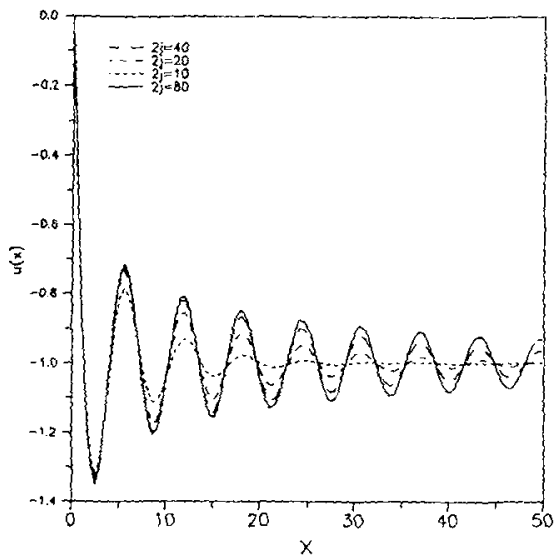

(c)

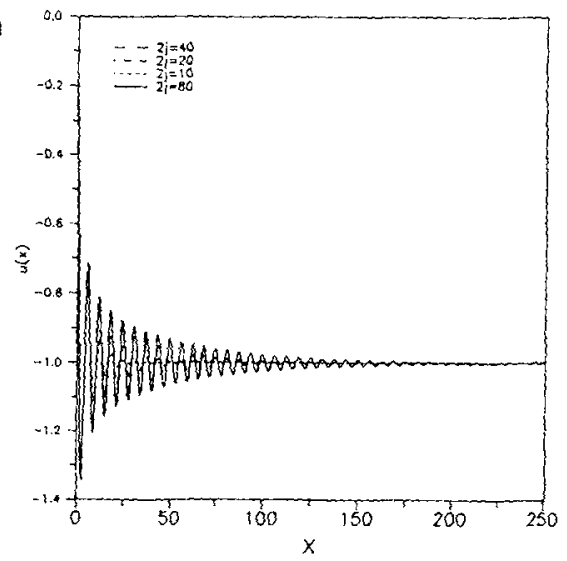

Fig. 3. HyperBurgers' shocks on the intervals (a) $x \in[0,15]$, (b) $[0,50]$ and (c) $[0,250]$. Solid: $j \approx 40$ (80-th derivative damping). Long dashes: $j \approx 20$. Short dashes: $j=10$. Dotted: $j=5$ (tenth-derivative damping). 
and similar expressions with the arguments of the trigonometric functions doubled when $j$ is odd. The similarity to Eqs. (2.9) and (2.10) is striking. In particular, the coefficient of the exponential decay, $\mu$, tends to zero with increasing $j$ so that the oscillations extend farther and farther from the front as observed.

Figure $3 \mathrm{a}$ also suggests there is a limiting form $u_{\infty}$ as $j \Rightarrow \infty$, which we will call the "infinity shock." For $x<4$, the four graphs are almost indistinguishable. For larger $x$, however, the $j=5$ curve is more and more rapidly damped and therefore is a poor approximation to the other curves. The $j=10$ graph, however, is a good approximation to both the first peak and trough of the graphs for larger $j$. The $j=20$ and $j=40$ curves are barely distinguishable on the whole interval $x \in[0,15]$. Only in Figs. $3 b$ and $3 \mathrm{c}$ do we see the $j=20$ shock damp out more rapidly than the $j=40$ solution so that the oscillations of the former have only half the amplitude of the latter at $x=50$.

The conclusion is that the portion of the infinity shock which would fit on Fig. 3a would differ little from the solid $(j=80)$ curve on that figure. As $j$ increases, $u_{j}(x)$ would approximate $u_{s c}(x)$ on a larger and larger $x$ interval.

This behavior with increasing $j$ is very much the same as we found via steepest descent for fronts of the hyperdiffusion equation in Sec. 2. To confirm the similarities, Fig. 4 is a graph of the sine coefficients for

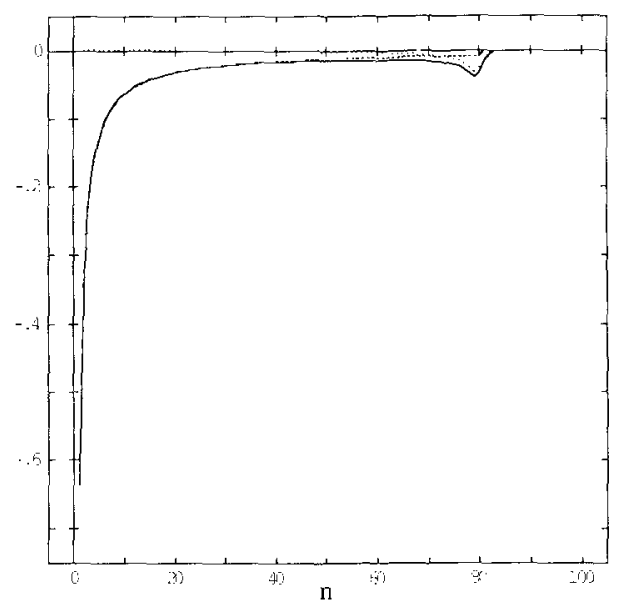

Fig. 4. Fourier sine coefficients for the $j=40$ hyperBurgers' shock: Solid: $b_{n}$ [coefficients of $u(x)$ ]. Dashed: $\beta_{n}$ [coefficients of $\left.-\operatorname{Si}(x)\right]$. Dotted: $\rho_{n}$ [coefficients of $\left.u(x)-(-\operatorname{Si}(x))\right]$. $L=80 / \pi$, so the coefficients of $\mathrm{Si}(x)$ are bandlimited to $n \leqslant 80$. 
$u_{40}(x), b_{n}$, together with the coefficients of the $x$-plus-sine approximation to the sine integral,

$$
\beta_{n} \equiv\left\{\begin{array}{ll}
-\frac{2}{n \pi} & n \leqslant \frac{L}{\pi} \\
0 & n>\frac{L}{\pi}
\end{array} \quad \text { Coefficients of “Gibbs' Shock”, Si }(x)\right]
$$

and the "residual" coefficients

$$
\rho_{n}=b_{n}-\beta_{n}
$$

We see that the residual coefficients are small in comparison to those of the Gibbs' Shock: the negative of $\mathrm{Si}(x)$ is a good first approximation to the $j=40$ hyperBurgers front.

However, it would be wrong to infer that the small difference between $u_{j}(x)$ and $-\mathrm{Si}(x)$ disappears in the limit $j \Rightarrow \infty$. First, note that the largest trough in $-\mathrm{Si}(x)$ is about -1.18 whereas the four converged graphs in Fig. 3a show that the corresponding trough in the infinity shock is about -1.34-roughly twice as large an oscillation. Second, Fig. 5 compares the residual coefficients defined by Eq. (4.2) for four different orders of damping, $j$.

Two trends are clear. One is the resemblance to $-\mathrm{Si}(x)$ already noted: As $j$ increases, the coefficients of degree $>80$ decrease very rapidly. This

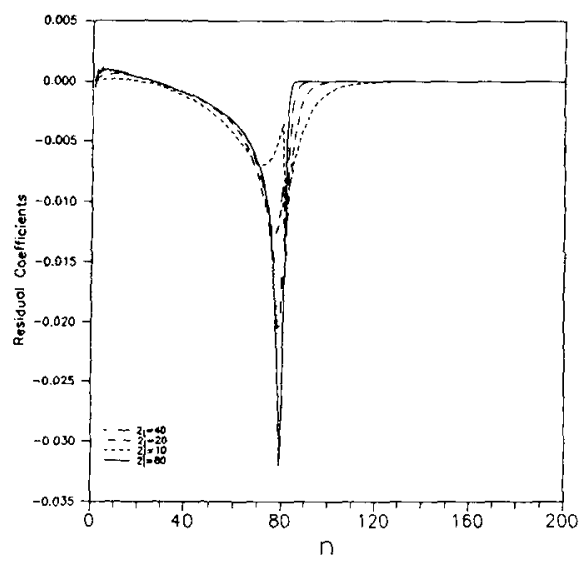

Fig. 5. Graph of the "residual" coefficients $\rho_{n}$ for $j=40$ (solid), $j=20$ (long dashes), $j=10$ (short dashes) and $j=5$ (dotted). The solid curve is the same as the dotted curve in Fig. 4 except that $y$-axis scale has been reduced by a factor of about 20: even the largest residual coefficient is small in comparison to $b_{1} \approx-2 / \pi$. 
strongly suggests that $u_{\infty}$, like $\operatorname{Si}(x)$, is a bandlimited function. The bandwidth condition $n \leqslant L / \pi$ for the discrete sine series translates into the statement that if the infinity shock is alternatively represented as a Fourier transform, then

$$
u_{\infty}(x)=\int_{-1}^{1} U_{\infty}(k) e^{i k x} d k
$$

for some function $U_{\infty}(k)$.

The second trend is that the residual coefficients have a taller and taller spike at the bandlimit, $n=L / \pi$, suggesting that $U_{\infty}(k)$ may contain the Dirac delta functions, $\delta(k-1)$ and $\delta(k+1)$. The spectral series for the $\operatorname{Si}(x)$ is free of delta functions.

Figure 6 displays the Fourier coefficients for the $(2 j)$-th derivative of $u(x)$ for $j=80$ - these may be equivalently interpreted as the coefficients of the nonlinear term, $u u_{x}$. Given that $u_{40}(x)$ is approximately bandlimited to $|k| \leqslant 1$ ( $n \leqslant 80$ in the graph), it is hardly surprising that the Fourier spectrum of the quadratically nonlinear term $u u_{x}$ is bandlimited to $k \leqslant 2$. What is remarkable is that (i) the spectral coefficients for $n<80(|k|<1)$ are very small, presumably zero in the limit and (ii) another apparent $\delta$-function at $k=1$ (and by symmetry, at $k=-1$ ).

Despite all these tantalizing clues, we have not been sufficiently clever to deduce an analytical form for the infinity shock: only that it rather resembles the corresponding Gibbs' Shock. Perhaps some reader will be smarter!

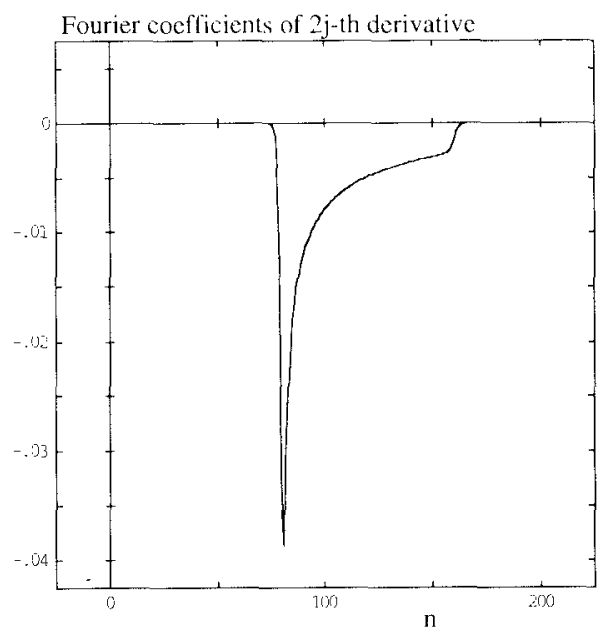

Fig. 6. Coefficients of the $2 j$ th derivative of $u(x)$ for $j=40$. (These are also the sine coefficients of $-u u_{\mathrm{x}}$.) 


\section{SPECTRAL VISCOSITY}

The hyperviscosities described earlier have one disadvantage when combined with spectral methods. When the damping order $j$ is fixed, the hyperviscosity coefficient must be $O\left(h^{2 j-1}\right)$ in order to smear out the front over the thickness of one grid spacing. (In this context, we mean that the crest and trough nearest the center of the front are separated by $O(h)$.) This unfortunately implies that the $O(1)$ wavenumbers will be artificially damped by $O\left(h^{2 j-1}\right)$. Thus, with $j=2$, the error in the $O(1)$ wavenumbers will decrease only cubically with $h$ even though the intrinsic accuracy of the spectral method decreases exponentially fast with $1 / h$. The obvious remedy is to choose a "spectral viscosity" which is exponentially rather than algebraically small for small wavenumbers.

As with other forms of artificial viscosity, the error is nonuniform: There are $O(1)$ "smearing" errors within one grid interval of the discontinuity in the exact solution. However, Tadmor $(1989,1990)$, Maday and Tadmor (1989), and Schochet (1991) have proved that the error is exponentially small outside the neighborhood of the front.

Their analysis does not demand a unique form for the artificial viscosity, but rather imposes some constraints, broad enough to describe a whole family of functional forms, which give spectral accuracy outside the frontal zone. These criteria are the following:

(i) The damping of small wavenumbers must be either identically zero or decrease exponentially with the Fourier truncation $N$.

(ii) The frontal zone should be as narrow as possible for a given $N$.

(iii) The oscillations of $u(x)$ in the frontal zone should damp out rapidly away from the center of the front.

(iv) The function $Q(k)$ should be infinitely differentiable for all real $k$ in the Fourier truncation where the damping term is of the form

$$
Q(k) U(k) \Leftrightarrow \int_{-\infty}^{\infty} q(y) u(x-y) d y
$$

where the left-hand expression is in wavenumber space whereas the right-hand side is the same expression in physical space; $U(k)$ and $Q(k)$ are the usual Fourier transforms of $u(x)$ and $q(x)$, respectively.

Tadmor (1989) experimented with a form which satisfies constraints (i)-(iii), but is not smooth because the first derivative of $Q(k)$ is discontinuous

$$
Q(k) \equiv \begin{cases}0 & k \leqslant m \\ -\varepsilon k^{2} & k>m\end{cases}
$$


where $m(N)$ is an increasing function of $N$, the truncation of the Fourier series, and $\varepsilon$ is a constant. As shown in Fig. 6.1, [Tadmor (1989)] this damping, although technically a "spectral" viscosity, gives very wiggly shocks as illustrated in our Fig. 7.

It is well known (Lighthiil, 1958) that the rate of decay of the Fourier transform of a function $q(x)$ as $|x| \Rightarrow \infty$ is inversely related to the smoothness of $Q(k)$, the function being transformed. In particular, if $Q(k)$ is a $C^{\infty}$ function, $q(x)$ will decay exponentially; this implies that the smearing of the discontinuity in the convolution integral will be a perturbation that will decay exponentially fast as we move away from the discontinuity. In other words, the effect of the damping is local and confined (except for an exponential tail) to the neighborhood of the front. Unfortunately, $Q(k)$ as defined by Eq. (5.2) has a jump discontinuity at $k=m$. This implies that its Fourier transform $q(x)$ will decay only as $O(1 /|x|)$ so that the damping term is a significant perturbation of $u(x)$ even at great distances from the discontinuity in $u(x)$.

This argument is a bit suspect as applied to Eq. (5.1) because $Q(k)$ must increase with $k$, and that implies that even if $Q(k)$ is $C^{\infty}$, its function

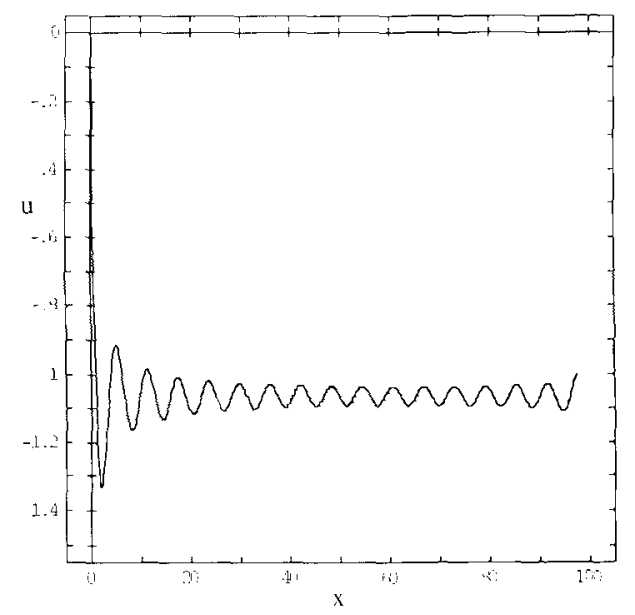

Fig. 7. The solution to the generalized hyperBurgers' equation when the viscosity is

$$
Q(k)= \begin{cases}0, & k \leqslant 1[n \leqslant 31] \\ -k^{2}, & k>1\end{cases}
$$

where the basis functions, periodic with a period of $62 \pi$, are $\sin (k x)$ with $k=n / 31$, $n=1,2, \ldots, 124$. Only half the period is shown because the solution is antisymmetric with respect to $x=0$. 
transform $q(x)$ exists only as a distribution. Nevertheless, the empirical evidence is that smoothness in $Q(k)$ is highly desirable.

Tadmor (1990) does not give the formula of his (smooth) spectral viscosity, so we experimented with a number of forms. Our best result is illustrated in Fig. 8 where

$$
Q(k)= \begin{cases}0, & \kappa<\frac{1}{4} \\ -\exp \left\{-\frac{1}{4\left|\kappa-\frac{1}{4}\right|}+16\left(\kappa-\frac{1}{4}\right)^{2}\right\}, & \kappa \geqslant \frac{1}{4}\end{cases}
$$

where $\kappa$ is the wavenumber scaled by the maximum wavenumber, i.e.,

$$
\kappa \equiv k / k_{\max }
$$

The $1 /|\kappa-1 / 4|$ in the exponential ensures that all derivatives of $Q(k)$ with respect to $k$ are 0 at the cutoff, $k=k_{\max } / 4$, thus ensuring continuity of the viscosity and all its $k$-derivatives, i.e. $Q(k)$ in Eq. (5.3) is a $C^{\infty}$ function.

The front is oscillatory, as Tadmor found for all the choices he tried. He obtained good results, but only after applying the postprocessing of Gottlieb and Tadmor (1985).

In order to compare the spectral viscosity with the solutions of the hyperBurgers Eq. (4.1), we normally used $k_{\max }=2$, which is equivalent to choosing the spatial period $P$ to be $\pi N$ where $N$ is the number of sines in

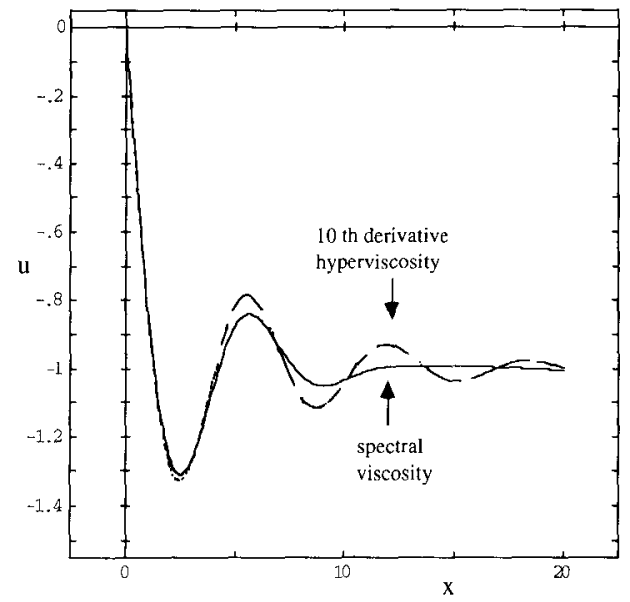

Fig. 8. Solid: the solution to the generalized Burgers equation $Q(k) U(k)+\mathscr{F}\left(u u_{x}\right)$ where $U(k)$ is the Fourier transform of $u(x), \mathscr{F}$ denotes the Fourier transform operation and $Q(k)$ is defined by Eq. (5.3). Dashed: the solution to the hyperBurgers Eq. (4.1) for $j=5$ (10th derivative damping). The spatial period is $160 \pi$ and $N=160$. 
the spectral basis. The reason for this choice is that for high order damping, the solution $u(x)$ to Eq. (4.1) is approximately bandlimited to $k<1$ since the damping, $-k^{2 j}$, increases very rapidly for larger $k$, annihilating the higher wavenumbers as previously illustrated in Figs. 4 and 5. The nonlinear term has double the bandwidth of $u(x)$ itself, so there is little error in truncating the spectral basis by ignoring $k>2$.

Figure 8 compares two solutions to the hyperBurgers equation: one with the spectral viscosity in Eq. (5.3) and the other with 10-th derivative damping. The spectral viscosity is a great improvement because the oscillations damp out much rapidly away from the origin than with hyperviscosity. Of course, the alternative of narrowing the oscillation zone by using a lower derivative damping, i.e., smaller $j$ in Eq. (4.1), is also available; for $j=1$ ( $2 d$ derivative damping), $u(x)$ varies monotonically. The price of smaller $j$ is stronger damping of small wavenumbers, however, and this is precisely what spectral viscosity aims to avoid.

One remarkable feature of the graph is that the first and deepest trough is almost identical for both solutions, even though the viscosities have rather different functional form as shown in Fig. 9. For larger $|x|$, the effects of different viscosities are very striking: the spectral viscosity solution damps out more quickly. For small $|x|$, however, there is a kind of universality. Perhaps this is not too surprising. Both cases have the same nonlinear term, $u u_{x}$, and both have viscosities which are large for $k>1$ and small for $k<1$ and equal 1 at $k=1$.

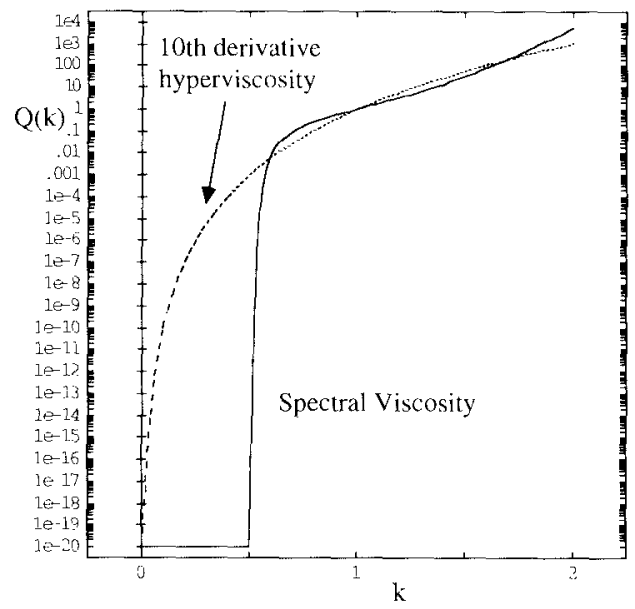

Fig. 9. A comparison of the absolute values of the viscosity coefficients, $Q(k)$, for the spectral viscosity Eq. (5.3) [solid] and for 10th derivative hyperviscosity [dashed] where $k_{\max }=2$. For the spectral viscosity $Q(k) \equiv 0$ for $k<1 / 2$. 
We found this common shape for the first trough - a minimum of about -1.33 at around $x=2.5$ - for many other choices of spectral viscosity. Indeed, a case which gave a minimum of -1.5 made us suspicious: Why was the minimum not -1.33 ? The answer was inadequate numerical resolution.

We must emphasize that this $33 \%$ overshoot is not completely universal. A second derivative damping, for example, gives a shock which is a hyperbolic tangent function and thus has no oscillations or overshoot at all. When the damping of the small wavenumbers is exponentially small, however, the shape of deepest trough seems to be the same across a wide range of functional forms for the viscosity. We have no explanation.

\section{SUMMARY}

In this work, we have used multiple scales perturbation analyses to reduce frontal dynamics to a stationary, generalized Burgers equation (Lesser and Chrighton, 1975, Blumen, 1991a, b, Boyd, 1992). We then solved this ordinary differential equation numerically to analyze the effects of different choices of viscosity on the structure of the fronts. By rescaling the length scale, the viscosity coefficient can be removed to give a canonical equation which is parameter-free except for the shape of the dependence of the damping on wavenumber.

Two numerical accomplishments are independent of shock phenomenology: (i) to illustrate the linear-plus-Fourier series pseudospectral method for computing tanh-like, nonperiodic solutions and (ii) to solve nonlinear differential equations of very high order.

Our other interesting conclusions are all tied to the physics of fronts. The first is that although shock-resolving algorithms are generally devised to enforce monotonic change across the front, we find that hyperviscosity and spectral viscosity generally lead to oscillatory frontal zones. It follows that we must be extremely careful in interpreting frontal oscillations in numerical models that use higher order damping. Such oscillations may not be a sign of numerical disaster, but rather a correct mathematical expression of the form of the dissipation. A second order viscosity is very special in generating a nonoscillatory, monotonic front.

A second conclusion is that using a hyperviscosity of very high order to resolve the front is a bad idea, even though the damping outside the frontal zone decreases rapidly with increasing order of dissipation. The reason is that the oscillations decay more and more slowly away from the center of the front as the dissipation order $j$ increases. Spectral viscosity is better because it gives exponentially small damping away from the front in com- 
bination with a narrow region of oscillation around the front (Tadmor, 1989, 1990, Maday and Tadmor, 1989, and Schochet, 1991).

A third conclusion, noted by Tadmor (1989) and explained here, is that it is important that the spectral viscosity be $C^{\infty}$, that is, a continuous function of wavenumber $k$ with continuous derivatives. A discontinuous viscosity may have little or no damping for small wavenumbers (good!), but the discontinuity (in wavenumber) will extend oscillations (in $x$ ) to vast distances from the center of the front.

A fourth conclusion is that there is a quasi-universality in the structure of the deepest trough corresponding to an overshoot of about $33 \%$ for a wide range of high order and spectral viscosities. The universality is limited because it is easy to find counterexamples; we computed a viscosity such that the sine integral $\mathrm{Si}(x)$, which has the usual Gibbs' overshoot of less than $18 \%$, is the exact solution. Nevertheless, it is striking the structure of the front is much more sensitive away from the front than in the trough nearest the center of the front.

Because hyperviscosities and spectral viscosities give oscillatory fronts, it is common to combine the artificial damping with a postprocessing filter such as that of Gottlieb and Tadmor (1985). Some schemes, such as Cai et al. (1989), explicitly fit a step function to the computed coefficients. This is successful as a running substitute for artificial viscosity, but would be disastrous if applied as a postprocessing to flows computed with hyperviscosity or spectral viscosity. The front illustrated in Fig. 8, for example, is not the sine integral, but rather has its own structure with almost twice the overshoot of $\operatorname{Si}(x)$.

The major open question is how to optimize the choice of spectral viscosity. The stationary generalized Burgers equation is a good starting point because the frontal structure has been reduced to an ordinary differential equation. It is simple in principle to solve this with a general viscosity coefficient, $Q(k)$ [in spectral space], and then optimize to find the best $Q(k)$ subject to various constraints. Unfortunately the constraints are multiple and messy.

One constraint is that the frontal zone should be as narrow as possible, that is, $u(x)$ should rapidly asymptote to -1 as $x \Rightarrow \infty$. A second constraint is monotonicity: real fronts are generally assumed to be free of the oscillations generated by a high order or spectral viscosity. (In many applications, the physical viscosity is so small that the physical fronts, whether oscillatory or not, have a width small in comparison to any reasonable grid spacing.) A third constraint is that the viscosity must taper the spectral coefficients so that the $N$ th coefficient is very small. Setting the viscosity identically equal to zero, for example, would make the solution of the hyperBurgers equation a step function, satisfying the first two constraints, 
but badly flunking the third constraint because of Gibbs oscillations. Good choices for spectral viscosity are now known, but determination of an optimum choice is an unsolved problem.

\section{ACKNOWLEDGMENTS}

This work was supported by the National Science Foundation through grants DMS8716766, OCE8800123, ECS0912263, and OCE911945 and by the Department of Energy through grant KC070101.

\section{APPENDIX. PERIODIC SHOCKS}

Fourier spectral methods are normally applied to compute solutions which are spatially periodic, as opposed to the infinite interval solutions calculated here. An obvious question is: how does the periodicity modify the shape of shocks? How relevant are the tanh-like solutions illustrated in Fig. 1 to the periodic, sawblade-like solutions of Tadmor (1989)?

The first answer, as expressed in Theorem 1, is that the tanh-like shocks are good local approximations to general shocks, regardless of the interval or the details of the initial conditions. This may be proved via the method of multiple scales (Boyd, 1992).

The second answer is that the tanh-like numerical solutions contain good global approximations to a simple class of spatial periodic nonlinear solutions. If we write

$$
u(x)=-x / L+f(x)
$$

as in Eq. (3.6) where $f(x)$ is periodic, then by direct substitution into the hyperBurgers' equation and a standard scale analysis,

$$
(-1)^{j} f_{2 j, x}+f f_{x}=0+O(1 / L) \quad f(x)=f(x+2 L)
$$

In words, the periodic part of our numerical solutions is itself a solution to the hyperBurgers' equation with an error $O(1 / L)$ where $L \gg 1$ is the spatial period. The graph of $f(x)$ qualitatively resembles the sawtooth-like solutions displayed in Tadmor $(1989,1990)$.

The second answer is really a reaffirmation of the first. The linear term $x / L$, which is the difference between the tanh-like $u(x)$ and the periodic $f(x)$, is a slowly-varying perturbation-"slowly-varying" denoting a length scale of $O(L)$. The method of multiple scales implies that the structure of the frontal zone must be the same for both the periodic solution and the tanh-like solution except for an error inversely proportional to the scale on which these solutions are diverging, $O(1 / L)$. 


\section{REFERENCES}

Abramowitz, M., and Stegun, 1. (1965). Handbook of Mathematical Functions. Dover, New York, p. 1000.

Bateman, H. (1915). Some recent researches on the motion of fluids. Mon. Wea. Rev. 43, 163-170.

Bender, C. M., and Orszag, S. A. (1978). Advanced Mathematical Methods for Scientists and Engineers. McGraw-Hill, New York, p. 266.

Benton, E. R., and Platzman, G.W. (1972). A table of solutions of the one-dimensional Burgers equation. Quart. Appl. Math. 30, 195-212.

Blumen, W. (1980). A comparison between the Hoskins-Bretherton model of frontogenesis and the analysis of an intense surface frontal zone. J. Atmos. Sci. 37, 64-77.

Blumen, W. (1981). The geostrophic coordinate transformation. J. Atmos. Sci. 38, 1100-1105.

Blumen, W. (1987). Energy spectra associated with Long's model for steady finite amplitude flow over orography. Geophys. Astrophys. Fluid Dyn. 38, 265-272.

Blumen, W. (1990a). Finite-amplitude mountain waves in a compressible atmosphere including the effects of dissipation. Geophys. Astrophys. Fluid Dynamics 52, 89-104.

Blumen, W. (1990b). A Semi-geostrophic Eady-wave frontal model incorporating momentum diffusion. Part I. Model and solutions. J. Atmos. Sci. 47, 2890-2902.

Blumen, W. (1990c). A Semi-geostrophic Eady-wave frontal model incorporating momentum diffusion. Part II. Kinetic energy and enstrophy dissipation. J. Atmos. Sci. 47, 2903-2908.

Boyd, J. P. (1980). The nonlinear equatorial Kelvin wave. J. Phys. Oceangr. 10, 1699-1718.

Boyd, J. P. (1982). The optimization of convergence for Chebyshev polynomial methods in an unbounded domain. J. Comp. Phys. 45, 43-79.

Boyd, J. P. (1988). Chebyshev domain truncation is inferior to Fourier domain truncation for solving problems on an infinite interval. J. Scient. Comp. 3, 109-120.

Boyd, J. P. (1989). Chebyshev and Fourier Spectral Methods. Lecture Notes in Engineering, Springer-Verlag. New York, 49, 800.

Boyd, J. P. (1992). The energy spectrum of fronts: The time evolution of shocks in Burgers' equation. J. Atmos. Sci. 49, 128-139.

Cai, W., and Gottlieb, D., and Shu, C.-W. 1989: Essentially nonoscillatory spectral Fourier methods for shock wave calculations. Math. Comp. 52, 389-410.

Charney, J. C. (1971). Geostrophic turbulence. J. Atmos. Sci. 28, 1087-1095.

Gottlieb, D., and Tadmor, E. (1985). Recovering pointwise values of discontinuous data with spectral accuracy, in: Progress and Supercomputing in Computational Fluid Dynamic's, E. M. Murman and S. S. Abarbanel, (eds.), Birkhauser, Basel, pp. 357-375.

Hedstrom, G. (1975). Models of difference schemes for $u_{t}+u_{x}=0$ by partial differential equations. Math. Comp. 29, 969-977.

Hooper, A. P., and Grimshaw, R. (1988). Travelling wave solutions of the KuramotoSivashinsky equation. Wave Motion 10, 405-420.

Hoskins, B. J., and Bretherton, F. P. (1972). Atmospheric frontogenesis models: Mathematical formulation and solution. J. Atmos. Sci. 29, 11-37.

Hoskins, B. J. (1982). The mathematical theory of frontogenesis. Ann. Rev. Fluid Mech. 14. $131-151$.

Kevorkian, J., and Cole. J. D. (1981). Perturbation Methods in Applied Mathematics. SpringerVerlag, New York, p. 558.

Korner, T. W. (1988). Fourier Analysis. Cambridge University Press, New York, p. 590.

Lesser, M. B., and Chrighton, D. G. (1975). Physical acoustics and the method of matched asymptotic expansions. W. P. Mason and R. N. Thurston, (eds.), Academic Press. New York, Phvsical Acoustics 11, 69-149. 
LeVeque, R. J. (1990). Numerical Methods for Conservation Laws. Birkhauser, Basel, pp. 117 121.

Lighthill, M. J. (1958). Introduction to Fourier Analysis and Generalized Functions. Cambridge University Press, Cambridge. p. 79.

Lyness, J. N. (1971). Adjusted forms of the Fourier Coefficient Asymptotic Expansion and applications in numerical quadrature. Math. Comp. 25, 87-104.

Lyness, J. N. (1984). The calculation of trigonometric Fourier coefficients. J. Comp. Phys. 54, $57-73$

Maday, Y., and Tadmor, E. (1989). Analysis of the spectral viscosity method for periodic conservation laws. SIAM J. Numer. Anal. 26, 854870.

Platzman, G. W. (1964). An exact integral of complete spectral equations for unsteady onedimensional flow. Tellts 16, 422-431.

Roe, P. L. (1986). Characteristic-based schemes for the Euler equations. Ann. Rev. Fluid Mech. 18. $337-365$.

Schochet, E. (1991). The rate of convergence of spectral-viscosity methods for periodic conservation laws. SIAM J. Numer. Anal., (to appear).

Tadmor, E. (1989). Convergence of spectral methods for nonlinear conservation laws. SIAM J. Numer. Anal. 26, 30-44.

Tadmor, E. (1990). Shock capturing by the spectral viscosity method. Comput. Meth. Appl. Mech. Engin. 80, 197-208.

Van Dyke, M. (1964). Perturbation Methods in Fluid Mechanics. Academic Press, New York, pp. 106-109.

Warming, R., and Hyett, ? (1974). The modified equation approach to the stability and accuracy analysis of finite difference methods. J. Comp. Phys. 14, 159-179.

Whitham, G. B. (1974), Linear and Nonlinear Waves. John Wiley, p. 99. 